\title{
Effect of current and daylight variations on small-pelagic fish aggregations (Selar crumenophthalmus) around a coastal fish aggregating device studied by fine-scale acoustic tracking
}

\author{
Manuela CAPELlo ${ }^{1, a}$, Marc Soria ${ }^{1}$, Gael Potin ${ }^{2}$, Pascal COTEL $^{1}$ and Laurent DAGorN ${ }^{3}$ \\ 1 UMR EME, Institut de Recherche pour le Développement (IRD), BP 50172, 97492 Sainte Clotilde Cedex, La Réunion, France \\ 2 ECOMAR, Laboratoire d'Ecologie marine, Université de La Réunion, Saint Denis, La Réunion, France \\ 3 UMR EME, Institut de Recherche pour le Développement (IRD), Victoria, Seychelles
}

Received 13 February 2012; Accepted 18 June 2012

\begin{abstract}
Several pelagic fish species are known to associate with floating objects. However, quantitative information on the main factors that drive this associative behaviour is still lacking. Small pelagic fish offer a particularly interesting case study for this phenomenon, since the small spatial scales involved in their association with floating objects allow experimental data to be collected at a fine scale. Here, we monitored twelve acoustically-tagged bigeye scads, Selar crumenophthalmus (Carangidae), (mean fork length $16.4 \mathrm{~cm}, \mathrm{SD} 2.1 \mathrm{~cm}$ ) around a floating object moored in shallow water (15 $\mathrm{m}$ depth) playing the role of a coastal fish aggregating device (FAD). To quantify the role played by variations in current and daylight, we calculated the speed distribution, pair correlation function and group polarization for the tagged fish hourly, from midday to dusk (13:00-18:00), for varying current strengths and daylight conditions. We found that the current induced a shift in the position of the aggregation upstream of the FAD, at distances that increased with the current strength. We found evidence of an expansion and a higher coordination in the aggregation at dusk, with increasing speed, distance among conspecifics and alignment. We discuss possible scenarios in which group polarization increases at dusk and suggest complementary measurements for future experiments that could confirm our findings.
\end{abstract}

Keywords: Acoustic telemetry / Small pelagic fish / Moored FAD / Fish aggregation / Behaviour / Daylight effects / Rheotaxis / Indian Ocean

\section{Introduction}

Some pelagic fish species are known to associate with objects floating at the surface of the ocean (Hunter and Mitchell 1967). Although this phenomenon is largely exploited by fishers, who use floating objects (usually called fish aggregating devices - FADs) to enhance their catch of various species (mainly tropical tunas), there is no scientific consensus on the origins of this behaviour (Fréon and Dagorn 2000; Castro et al. 2002). With the increasing use of drifting FADs by industrial purse seiners all over the world, the scientific community has questioned the effects of FADs on the behaviour of fish (Marsac et al. 2000; Hallier and Gaertner 2008). So far, most behavioural studies have been conducted on tropical tunas mainly Thunnus albacares, but also Katsuwonus pelamis and Thunnus obesus (Dempster and Taquet 2004) and their movements within FAD arrays (Klimley and Holloway 1999; Ohta and Kakuma 2005, Dagorn et al. 2007). However, studies on smaller species around shallow coastal FADs (Soria et al. 2009; Capello et al. 2011) have recently revealed the

a Corresponding author: manuela.capello@ird.fr potential of using fine-scale data (often difficult to obtain on larger species in the open ocean) to understand behavioural processes.

Fish aggregations could depend on several factors, such as the intrinsic characteristics of the floating object, oceanographic characteristics or the abundance of prey, predators or conspecifics. The problem is complex due to the high likelihood of confounding these multiple factors and the difficulty of conducting accurate field-based measurements capable of quantifying them. Using fine-scale acoustic tagging data on a small pelagic fish around a single FAD, a recent study quantified the main factors shaping the zone of aggregation for bigeye scads, Selar crumenophthalmus (Carangidae) (Capello et al. 2011). Results showed that the degree of sociability of individuals, as well as their swimming speed, strongly affects the radius of the aggregation. These findings came from observations in a 1-hour temporal window, during which the variability of the environmental conditions were negligible. Nevertheless, external factors, such as current and daylight variation, could affect the behaviour of fish when they were associated with a FAD. Although empirical observations made by fishers 
assessed the importance of these quantities (in particular the effect of current on the position of fish in relation to the floating object) and sun-synchronous space-use patterns have been recently identified in other species (Hitt et al. 2011), so far no studies have been able to quantify their effects on fish aggregations around FADs.

The bigeye scad is a small coastal pelagic fish, common in tropical and subtropical waters. Around La Reunion Island, bigeye scads are very abundant in the north western waters, where they approach the coastline in the period from March to October (Roos et al. 2007). Previous studies showed that bigeye scads mainly feed during the night (Roux and Conand 2000) and are associated with FADs during the day (Soria et al. 2006; Capello et al. 2012). In this paper, we investigated the effects of current and daylight variation on the behaviour of bigeye scads associated with a floating object. Experiments were conducted during the daytime and were designed to examine associative behaviour with respect to fine-scale environmental changes.

\section{Materials and methods}

\subsection{Experimental setting}

Experiments were conducted in Saint Paul's bay $\left(21^{\circ} 00^{\prime} \mathrm{S}\right.$, $55^{\circ} 16^{\prime}$ E), Reunion Island (South Western Indian Ocean). Forty bigeye scads (mean fork length $16.4 \mathrm{~cm}$, SD $2.1 \mathrm{~cm}$ ) were caught using hand lines, transported in baskets and maintained in tanks at the Aquarium de La Reunion for ten days to acclimate. They were fed, and treated with a solution of methylene blue and copper sulfate to kill bacteria and prevent the growth of fungi. The tagging operation was carried out on the 1 May 2003. We retrieved twelve individuals from the tank and gastrically implanted them with acoustic tags by ingurgitation. The HTI ${ }^{\mathrm{TM}}$ acoustic tags (Model 795) used are $7 \mathrm{~mm}$ diameter, $17 \mathrm{~mm}$ length and weigh $1.5 \mathrm{~g}$ in water. This weight was less than $0.1 \%$ of the mean fish weight and did not affect their buoyancy (Almeida et al. 2007). In situ tests led us to choose a pulse duration of $4 \mathrm{~ms}$. The tag repetition rate, which was programmed to be different for each tagged fish, ranged between 1.43 and $1.16 \mathrm{~s}^{-1}$.

The HTI ${ }^{\mathrm{TM}}$ Acoustic Tag Tracking System (Model 290) set up was composed of five hydrophones connected by cables to the Acoustic Tag Receivers system, which was installed on a boat of $12 \mathrm{~m}$ length and fixed at $17 \mathrm{~m}$ depth by five anchors to prevent any movement. Four hydrophones were positioned around the boat in a square of approximately $100 \mathrm{~m}$ width.

The fifth hydrophone was located in the centre of the square. The cables connecting the hydrophones to the boat formed a vertical submerged structure whose position was taken as our FAD position. With this set-up, a signal emitted by the tag within the square of detection was received by the different hydrophones and the reception time recorded. The difference in detection times among the receivers was then used to estimate the tag location through triangulation, see details in Capello et al. (2011).

The twelve tagged fish were released on 3 May 2003 at 12:00 in the vicinity of the boat, anchored in Saint Paul's Bay, in the vicinity of the fishing location. Because bigeye scad is an obligate schooling fish species (Soria et al. 2007), we released the 12 tagged fish with 28 non-tagged conspecifics so they could immediately form small schools. The 3D tracking of each tagged fish (one position every second with sub-metre resolution) was possible within a radius of approximately $50 \mathrm{~m}$ from the FAD. All tagged fish stayed within the zone of detection until 19:00, with very few excursions outside the range of detection.

At the time the experiment was conducted, sunset was at 17:30. Current was measured using an Aanderaa RMC 9 Self Recording Current Meter, which was fixed under the boat (at $5 \mathrm{~m}$ depth) in order to record the horizontal current speed and direction.

More details on the experimental setting can be found in Capello et al. (2011).

\subsection{Methods for data processing and analysis}

Data processing involved two steps. First, the acoustic record of each tag on each of the five hydrophones was manually checked using $\mathrm{HTI}^{\mathrm{TM}}$ Mark Tags Software to exclude acoustic noise. Second, files were processed in HTI ${ }^{\mathrm{TM}}$ Acoustic Tag program to track acoustic echoes and calculate fish positions through triangulation. The accuracy of the estimated position of fish in the monitoring network ranged from 0.1 to $0.3 \mathrm{~m}$ the horizontal plane. In the vertical direction, the accuracy was lower (around $1 \mathrm{~m}$ ).

Due to the system geometry, where the FAD was associated with a submerged vertical structure reaching the seabottom, data analysis focused on the $x y$ plane, neglecting the vertical dimension. This approach was supported by previous acoustic survey measurements (Josse et al. 2000; Doray et al. 2006; Moreno et al. 2007), where the fish spatial distribution along $z$ was not affected by the presence of the FAD but instead depended on the fish species.

Because we had detailed spatial information concerning several individuals, we calculated the pair-distribution function $g(r)$ (Cavagna et al. 2008, 2010) among synchronous fish. This quantity gives information on the probability of finding a pair of individuals at distance $r$ from each other. We took synchronicity intervals of 1 second because this time frame was sufficient to observe a large number of synchronous fish. We considered the time-averaged fish pair-correlation function $g(r)$ at different hours of the day, which can be written as:

$$
g(r)=\left\langle\frac{1}{N(t)} \frac{\sum_{i j} \delta\left(r-r_{i j}(t)\right)}{2 \pi r d r}\right\rangle,
$$

where $N(t)$ is the number of coplanar pairs of fish detected in the temporal interval of one second $[t, t+1 s]$ and $r_{i j}(t)$ is the planar distance among synchronous fish $i$ and $j$. Coplanarity was established when two fish were within $1 \mathrm{~m}$ in the $z$ direction, according to our experimental accuracy in the vertical direction. The delta function selects fish pairs at planar distance in the range $[r-d r, r]$, with $d r=0.3 \mathrm{~m}$, and the brackets denote the time average over one hour. 

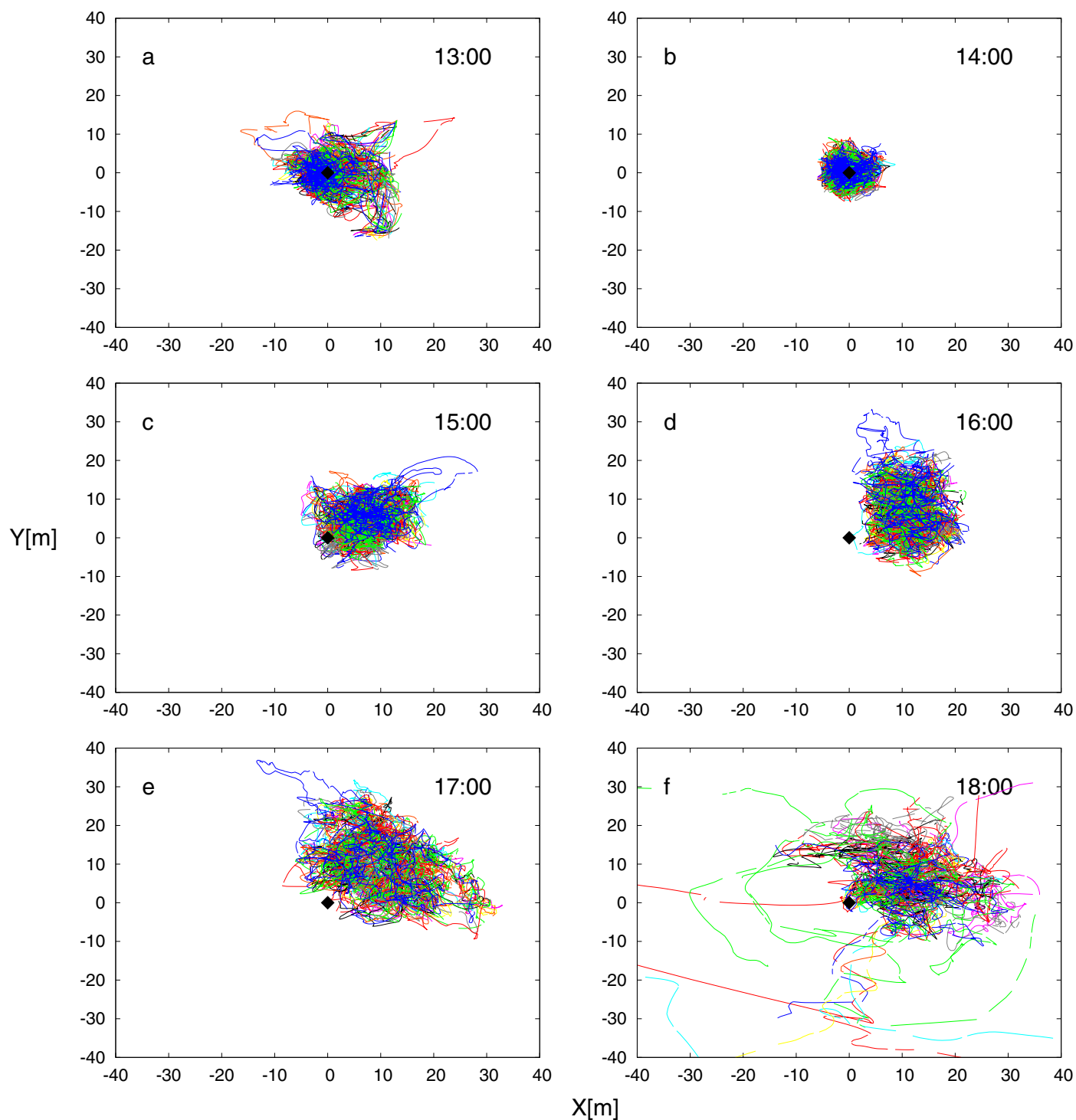

Fig. 1. Fish trajectories in the $x y$ plane at different hours of the day, from 13:00 (a) to 18:00 (f). Each colour represents a different fish. The black diamond corresponds to the position of the FAD.

Another quantity that we calculated was the tagged-fish group polarization in the $x y$ plane:

$$
\phi=\left\langle\left|\frac{1}{N(t)} \sum_{i} \frac{\boldsymbol{v}_{i}}{\left|\boldsymbol{v}_{i}\right|}\right|\right\rangle,
$$

where the sum runs over the $N(t)$ fish detected in the temporal interval $[t, t+1 s], \boldsymbol{v}_{i}$ is the planar speed vector of fish $i$ and $\left|\boldsymbol{v}_{i}\right|$ denotes its modulus and the brackets denote the time average over one hour. This quantity provides information about the degree of alignment among individuals in the group and is equal to 1 if all fish swim in the same direction in the $x y$ plane.

Finally, we characterized the individual fish dynamics through the calculation of the swimming speed (ground speed) and the turning angle distribution at the different hours.

For the following analysis, we denoted the time intervals of one hour h:min (for example 13:00 refers to the time interval between 13:00 and 14:00). We compared the swimming speed and turning angle distributions at subsequent hours using two-sample Kolmogorov-Smirnov tests (R Development Core Team 2011), testing the null hypothesis of equality between two distributions. Moreover, we used the same test to compare the distribution of fish-group polarization at subsequent hours, testing the null hypothesis that the cumulative density function of the group polarization at $h+1: \min$ was larger than at h:min.

\section{Results}

The fish trajectories in the $x y$ plane showed clear differences according to the hour of the day (Fig. 1). In order to characterize this variability, we calculated the position of the tagged-fish centre of mass and compared it with the average value of the current strength for each hour, see Fig. 2. We 

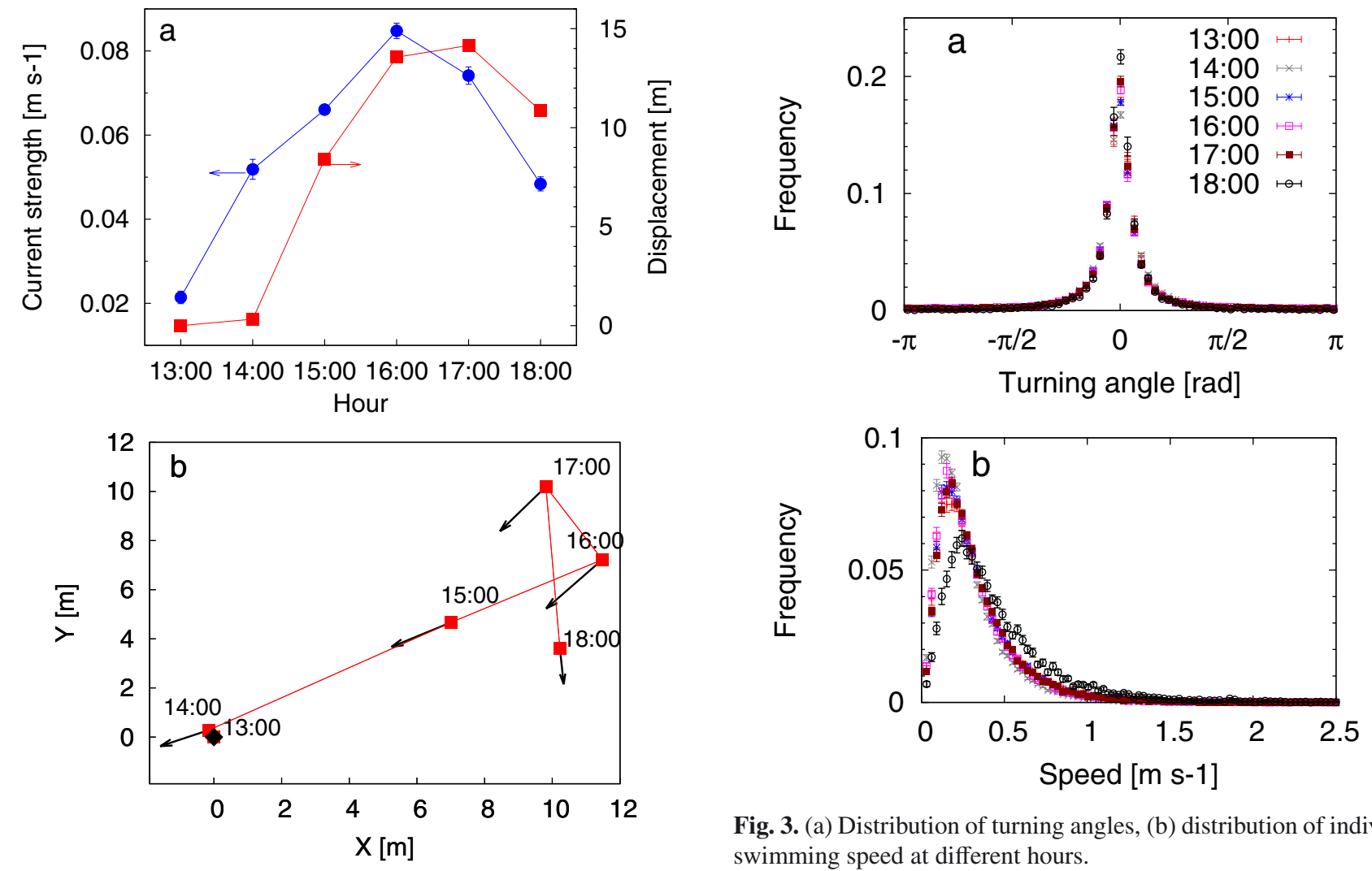

Fig. 3. (a) Distribution of turning angles, (b) distribution of individual swimming speed at different hours.

Fig. 2. (a) Mean centre of mass displacement (squares, right axis) and average value of the current (circles, left axis), calculated over time intervals of 1 hour, from 13:00 to 18:00. Bars indicate the standard error of the mean; (b) position of the fish centre of mass in the $x y$ plane at different hours of the day. Arrows indicate the current direction, with their length being proportional to the current strength. The black diamond indicates the FAD position.

found that higher (or lower) current strengths led to increasing (or decreasing) distances between the tagged fish and the FAD (Fig. 2a), in the direction upstream of the FAD (Fig. 2b). However, the increase of the current strength at 14:00 did not reflect a significant displacement of the tagged-fish centre of mass, until 15:00.

For all hours, the pair correlation $g(r)$ (Eq. (1)) showed a maximum around $0.6+/-0.3 \mathrm{~m}$, signalling that the most frequent fish-fish distance was always at short range (see inset of Fig. 4). The shape of the pair-distribution at 14:00 was particularly notable, as the group of tagged fish showed a higher cohesion (Fig. 4). Conversely, at late hours, fish tended to occupy larger distances, manifested by longer tails in $g(r)$ later in the day. This trend was smooth from 15:00 to 18:00, indicating a gradual expansion of the aggregation with time.

Finally, the distribution of the tagged-fish group polarization showed an enhancement of fish alignment at late hours, characterized by a smooth, significant shift of the polarization towards higher values from 15:00 to 18:00 ( $p$-value $>0.05$ ), see Fig. 5.

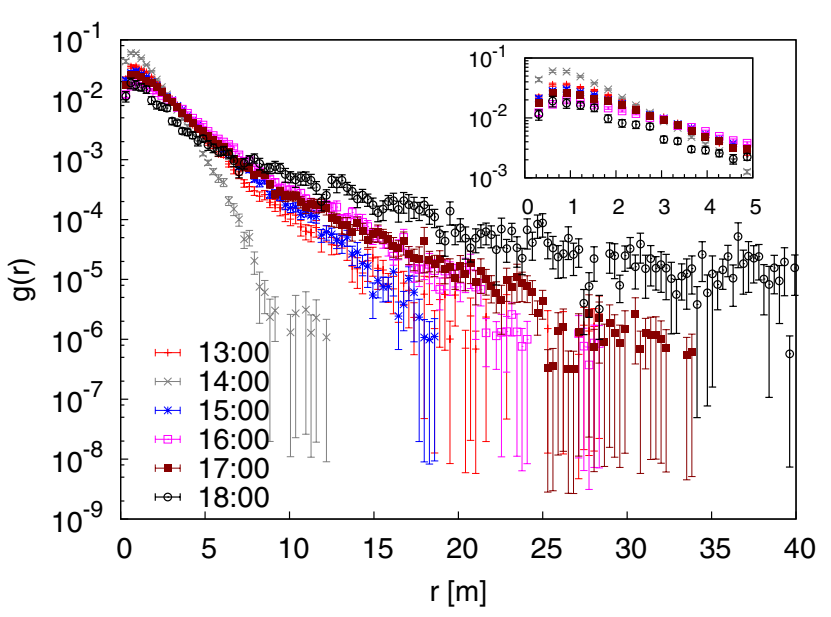

Fig. 4. Fish pair-distribution function (Eq. (1)) at different hours. Inset: zoom of $g(r)$ at small $r$.

\section{Discussion}

The above results clearly indicate that the fine-scale information obtained through the HTI acoustic technique is very promising for characterizing the role of different factors affecting the shape of small-pelagic fish aggregations around a FAD. Indeed, we could evaluate the effect of current variations on the position of the aggregation through the quantification of the 


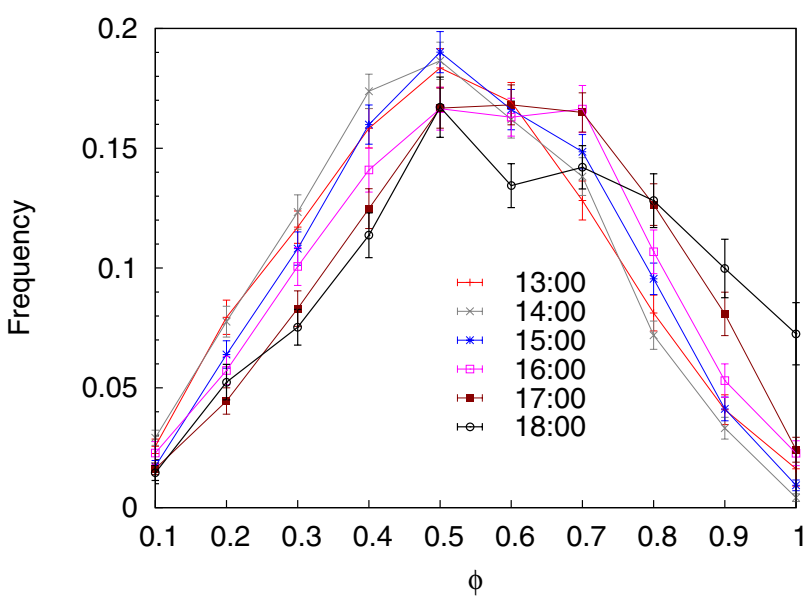

Fig. 5. Distribution of the tagged-fish polarization (Eq. (2)) at different hours.

displacement of the tagged-fish centre of mass with respect to the location of the FAD. The effect of current on fish aggregation was clear, with the group moving upstream of the FAD at large distances when the current increased. According to Lindquist and Pietrafesa (1989), one possible reason for this orientation may be that the fish were choosing the location where they would likely find the least amount of current flow and would therefore have to spend the least amount of energy swimming in the current. This location could correspond to the up-current side of the FAD, where incoming current flow is deflected back upon itself, resulting in narrow elliptical eddies that tend to negate current flow. A supporting argument, mentioned in Lindquist and Pietrafesa (1989), is the advantage of facing into currents that bring food into the reef. Food that the fish miss on the first pass may be dragged into the up-current vortex reversal, thus giving the bigeye scads to opportunity to feed on several passes of the food organisms. Additionally, we observed a delay of about one hour in fish response to the current around 14:00. At this time, other anomalies were also observed, like a contraction of the aggregate shown by the fish pair distribution. This might signal that an unobserved event, like the presence of a predator or the arrival of another group of fish, occurred around 14:00, inducing a group contraction and localization to the FAD that was much stronger than expected. In a similar way, we could not assess the origin of the increase in fish speed at 18:00, where possible unobserved predation effects might have affected the behaviour of the tagged fish.

The effect of daylight emerged from the smooth variation of the tagged-fish pair-distribution and polarization observed towards dusk. These changes could not be related to current effects, since the current strength and angle were nearly the same around 15:00 and 17:00. One could argue that these observations were the consequence of a gradual displacement of fish from their release point that happened to coincide with approaching dusk. However, our data instead indicate a "group dispersion", i.e., fish stayed further from each other (Fig. 4), rather than a dispersion from the release point, since the centre of mass distance from the FAD (the release point) was nearly constant from 16:00 to 18:00 (Fig. 1). This pattern, where fish aggregations are characterized by low packing densities at night, is well known from acoustic measurements (Fréon et al. 1996).

Additionally, we found that the polarization of the group of tagged fish increased smoothly towards dusk, confirming that vision alone could not mediate fish alignment. Both lateral line and vision are employed simultaneously to maintain group polarization (Pitcher et al. 1976). These results were quite surprising, however, since we expected the opposite scenario: with a gradual expansion in the fish aggregation inducing a lower polarization of the group. It is possible that other factors, not measured in this experiment, could be crucial in explaining these findings. For example, the number of conspecifics present in the aggregation could have increased from noon to dusk. Since we only quantified the tagged-fish behaviour, we observed a consequent expansion in the aggregation. In this case, the fish aggregation would increase in size over the course of the day and manifest a smooth transition towards higher degrees of polarization at dusk, before fish departure. Another possible explanation could be that group polarization depends on distance from the FAD. In this scenario, the closer the group is to the FAD, the more the thigmotaxis towards the FAD perturbs the alignment among conspecifics. In this way, the polarization of aggregated fish increases when the group moves away from the FAD, up to the fully-polarized limit of free schools.

While previous studies have shown that social effects and speed (Capello et al. 2011) play a fundamental role in the aggregations of small pelagic fish around FADs, we demonstrated that variable environmental conditions could strongly affect the properties of the aggregation. However, although we showed that changes in current and daylight can play a role, these factors alone cannot explain the full dynamics of the system observed during the day. Possible inter-specific interactions and predation effects, as well as an increasing number of conspecifics, might be important in determining the properties of the aggregation. Further investigations based on similar experimental settings, complemented with continuous visual inspections near the FAD, could confirm our picture of the aggregation dynamics.

Acknowledgements. We thank M. Taquet, L. Bigot, P. Durville, M. Timko, G. Fritsh for their help with the fishing and tagging operations and P. Fréon for interesting discussions. This work was supported by the Regional Council of La Reunion Island, the French Ministry of Overseas Regions, the "Run Sea Science" (Theme Capacity Building) and the "MADE: Mitigating adverse ecological impacts of open ocean fisheries" (Theme 2 - Food, Agriculture, Fisheries and Biotechnology, contract \#210496) FP7 European projects.

\section{References}

Almeida P., Quintella B., Costa M., Moore A., 2007, Developments in Fish Telemetry. Proc. the 6th Conference on Fish Telemetry held in Europe. Springer-Verlag New York.

Capello M., Soria M., Cotel P., Deneubourg J.L., Dagorn L., 2011, Quantifying the interplay between environmental and social effects on aggregated-fish dynamics. PLoS One 6(12): e28109. 
Capello M., Soria M., Cotel P., Potin G., Dagorn L., 2012, The heterogeneous spatial and temporal patterns of behavior of small pelagic fish in an array of Fish Aggregating Devices (FADs). J. Exp. Mar. Biol. Ecol. (in press).

Castro J.J., Santiago J.A., Santana-Ortega A.T., 2002, A general theory on fish aggregation to floating objects: an alternative to the meeting point hypothesis. Rev. Fish Biol. Fish. 11, 255-277.

Cavagna A., Cimarelli A., Giardina I., Orlandi A., Parisi G., et al., 2008, New statistical tools for analyzing the structure of animal groups. Math. Biosci. 214, 32-37.

Cavagna, A., Cimarelli A., Giardina I., Parisi G., Santagati R., Stefanini F., Tavarone R., 2010, From empirical data to interindividual interactions: unveiling the rules of collective animal behaviour. Math. Models Methods Appl. Sci. 20, 1491-1510.

Dagorn L., Holland K.N., Itano D.G., 2007, Behavior of yellowfin (Thunnus albacares) and bigeye (T. obesus) tuna in a network of fish aggregating devices (FAD). Mar. Biol. 151, 595-606.

Dempster T., Taquet M., 2004, Fish aggregation device (FAD) research: gaps in current knowledge and future directions for ecological studies. Rev. Fish Biol. Fish. 14, 21-42.

Doray M., Josse E., Gervain P., Reynal L., Chantrel J., 2006, Acoustic characterisation of pelagic fish aggregations around moored fish aggregating devices in Martinique (Lesser Antilles). Fish. Res. $82,162-175$.

Fréon P., Gerlotto F., Soria M., 1996, Diel variability of school structure with special attention on transition periods. ICES J. Mar. Sci. 53, 459-464.

Fréon, P., Dagorn, L., 2000, Review of fish associative behaviour: toward a generalisation of the meeting point hypothesis. Rev. Fish Biol. Fish. 10, 183-207.

Hallier J.P., Gartner D., 2008, Drifting fish aggregation devices could act as an ecological trap for tropical tuna species. Mar. Ecol. Prog. Ser. 353, 255-264.

Hitt S., Pittman S.J., Brown K. A., 2011, Tracking and mapping sunsynchronous migrations and diel space use patterns of Haemulon sciurus and Lutjanus apodus in the US Virgin Islands. Environ. Biol. Fish 92, 525-538.

Hunter J.R., Mitchell C.T., 1967, Field experiments on the attraction of fish to floating objects. J. Cons. Perm. Int. Explor. Mer 31, 427-434.
Josse E., Dagorn L., Bertrand A., 2000, Typology and behaviour of tuna aggregations around fish aggregating devices from acoustic surveys in French Polynesia. Aquat. Living Resour. 13, 183-192.

Klimley A.P., Holloway C.F., 1999, School fidelity and homing synchronicity of yellowfin tuna, Thunnus albacares. Mar. Biol. 133, 307-317.

Lindquist D.G., Pietrafesa L.J., 1989, Current vortices and fish aggregations: the current field and associated fishes around a tugboat wreck in Onslow Bay, North Carolina. Bull. Mar. Sci. 44, 533-544.

Marsac F., Fonteneau A., Ménard F., 2000, Drifting FADs used in tuna fisheries: an ecological trap? In: Pêche thonière et dispositifs de concentration de poissons. Le Gall J.-Y., P. Cayré, M. Taquet (eds). Ed. Ifremer, Actes Colloq. 28, 537-552.

Moreno G., Josse E., Brehmer P., Nøttestad L., 2007, Echotrace classification and spatial distribution of pelagic fish aggregations around drifting fish aggregating devices (DFAD). Aquat. Living Resour. 20, 343-356.

Ohta I., Kakuma S., 2005, Periodic behavior and residence time of yellowfin and bigeye tuna associated with fish aggregating devices around Okinawa Islands, as identified with automated listening stations. Mar. Biol. 146, 581-594.

Pitcher T.J., Partridge B.L., Wardle C.S., 1976, A blind fish can school. Science 194, 963-965.

R Development Core Team, 2011, R: A language and environment for statistical computing. R Foundation for Statistical Computing, Vienna, Austria. ISBN 3-900051-07-0, URL http://www.R-project.org/.

Roos D., Roux O., Conand F., 2007, Notes on the biology of the bigeye scad, Selar crumenophthalmus (Carangidae) around Reunion Island, southwest Indian Ocean. Sci. Mar. 71, 137-144.

Roux, O., Conand, F., 2000, Feeding habits of the bigeye scad, Selar crumenophthalmus (Carangidae), in La Réunion Island waters (south-western Indian Ocean). Cybium 24, 173-179.

Soria M., Fréon P., Chabanet P., 2007, Schooling properties of an obligate and a facultative fish species. J. Fish Biol. 71, 1257-1269.

Soria M., Dagorn L., Potin G., Fréon P., 2009, First field-based experiment supporting the meeting point hypothesis for schooling in pelagic fish. Anim. Behav. 78, 1441-1446. 